\title{
Breastfeeding self-efficacy and breastmilk feeding for moderate and late preterm infants in the Family Integrated Care trial: a mixed methods protocol
}

\author{
Meredith Brockway ${ }^{1 *}$ (D), Karen M. Benzies ${ }^{1}$, Eloise Carr $^{1}$ and Khalid Aziz²
}

\begin{abstract}
Background: Breastmilk is the ideal nutrition for preterm infants. Yet, breastmilk feeding rates among preterm infants are substantially lower than those of full-term infants. Barriers incurred through hospital care practices as well as the physical environment of the neonatal intensive care unit (NICU) can result in physical and emotional separation of infants from their parents, posing a substantial risk to establishing and maintaining breastfeeding. Additionally, current practitioner-focused care provision in the NICU can result in decreased breastfeeding selfefficacy (BSE), which is predictive of breastfeeding rates in mothers of preterm infants at 6 weeks postpartum.
\end{abstract}

Methods: Family Integrated Care (FICare) integrates and supports parents to actively participate in the care of their infant while in the NICU. Nested within the broader FICare trial, we will conduct an explanatory sequential mixed methods study to investigate if FICare improves maternal BSE and rates of breastmilk feeding in moderate and late preterm infants at discharge from the NICU. In phase 1, we will calculate the mean difference between admission and discharge BSE scores for the intervention group. Mothers who score in the top and bottom 20th percentile of change scores will be invited to participate in a semi-structured telephone interview exploring maternal experiences with infant feeding in the NICU. We will conduct inductive thematic analysis to identify and describe the facilitators and barriers of FICare on maternal feeding experiences. Once data saturation is achieved and themes have been established, phase 2 will revisit the quantitative data to determine whether FICare was impactful on BSE and breastmilk feeding rates. Findings from the qualitative and quantitative phases will be integrated to determine how infant feeding experiences on FICare units work to improve or detract from maternal BSE and rates of breastmilk feeding.

Discussion: FICare may help to improve maternal BSE and rates of breastmilk feeding in moderate and late preterm infants. Improved breastmilk feeding outcomes can have a substantial impact on overall infant health, developmental outcomes, and maternal-infant bonding and will help to improve long-term health outcomes for moderate and late preterm infants.

Trial registration: (NCT02879799). Registered May 27, 2016 protocol version June 9, 2016 Version 2.

Keywords: Breastmilk feeding, Breastfeeding, Breastfeeding self-efficacy, Mixed-methods, Thematic analysis

\footnotetext{
* Correspondence: mbrockwa@ucalgary.ca

${ }^{1}$ Faculty of Nursing, University of Calgary, Calgary, AB, Canada

Full list of author information is available at the end of the article
}

(c) The Author(s). 2018 Open Access This article is distributed under the terms of the Creative Commons Attribution 4.0 International License (http://creativecommons.org/licenses/by/4.0/) which permits unrestricted use, distribution, and reproduction in any medium, provided you give appropriate credit to the original author(s) and the source, provide a link to the Creative Commons license, and indicate if changes were made. The Creative Commons Public Domain Dedication waiver (http://creativecommons.org/publicdomain/zero/1.0/) applies to the data made available in this article, unless otherwise stated. 


\section{Background}

Globally, preterm birth rates (born prior to 37 weeks gestational age [GA]) range from 5 to $18 \%[1,2]$. Over $80 \%$ of preterm infants are born moderate (32 weeks and zero days $\left[32^{0 / 7}\right]$ to $33^{6 / 7}$ weeks GA) or late $\left(34^{0 / 7}\right.$ weeks to $36^{6 / 7}$ weeks GA) preterm [3, 4]. Prematurity is a significant contributor to child morbidity and a primary concern for child health clinicians $[1,2,4,5]$. Although not as medically complex as their early preterm (born prior to 32 weeks GA) counterparts, moderate and late preterm infants are at risk for several health and developmental issues [6], and often require level II neonatal intensive care [7]. Appropriate nutrition beginning at birth is a key component to lifelong health [8] and breastmilk feeding is the recommended optimum feeding method for preterm infants [9-12]. However, breastmilk feeding rates among preterm infants are substantially lower than those of full-term infants [13, 14]. Moderate and late preterm infants may have poor feeding skills that limit breastmilk intake and jeopardize infant growth and development [6]. The physical environment of the neonatal intensive care unit (NICU), and practices that physically and emotionally separate infants from their mothers, pose a risk to establishing and maintaining breastmilk feeding [15]. Despite recommendations for family centered care [16-18], the traditional model of care in NICUs situates healthcare professionals as the primary care provider. Frequently, mothers are relegated to the role of supplementary care provider or observer [18], which may limit time spent with their infant(s) and educational opportunities [19]. The traditional model of care can result in feelings of parental detachment, ineffective parenting, parenting stress, and loss of control [19, 20]. Further, traditional models of care decrease parenting and breastfeeding self-efficacy, potentially contributing to lower breastmilk feeding rates [15]. Integrating mothers into the care of their infants in the NICU may improve maternal breastfeeding self-efficacy and increase rates of breastmilk feeding at discharge.

\section{Breastfeeding self-efficacy}

Breastfeeding self-efficacy is a social cognitive theory adapted by Dennis [21]. Breastfeeding self-efficacy captures how a mother perceives her ability to breastfeed rather than her actual ability to succeed at breastfeeding [21-23]. Mothers with high self-efficacy are often able to overcome barriers that those with low self-efficacy would find overwhelming [24]. Breastfeeding self-efficacy is informed by four sources of information: (i) performance accomplishments, (ii) vicarious experience of seeing other mothers breastfeed, (iii) verbal persuasion by influential others, and (iv) the mother's physiological/affective state $[21,25]$. Breastfeeding self-efficacy can predict breastfeeding outcomes at 1 and 2 months postpartum in mothers of full-term infants [26] and it is a modifiable factor that can influence breastfeeding success [20, 2628]. Few studies have been conducted using breastfeeding self-efficacy theory in mothers of preterm infants $[20,29]$. Interventions to improve breastfeeding self-efficacy may improve breastmilk feeding rates and subsequent health outcomes for moderate and late preterm infants.

\section{Study aim}

This study is nested within a larger cluster randomized control trial (cRCT) assessing multiple outcomes of a Family Integrated Care (FICare) for moderate and late preterm infants in level II NICUs [30]. The primary outcome of the FICare cRCT is to evaluate the effect of FICare on length of stay in the level II NICU. The aim of the present study is to determine if FICare improves maternal breastfeeding self-efficacy and resultant breastmilk feeding rates in mothers of moderate and late preterm infants who were admitted to a level II NICU. The specific objectives are:

i. To determine if FICare is effective in improving breastfeeding self-efficacy in mothers of moderate and late preterm infants between admission to and discharge from a level II NICU.

ii. To determine if FICare is effective in increasing breastmilk feeding rates in mothers of moderate and late preterm infants at discharge from the NICU.

iii. To explore maternal experiences with infant feeding while admitted to the NICU.

iv. To determine if or how maternal experiences with infant feeding work to inform maternal breastmilk feeding rates while in the FICare NICU environment.

\section{Methods}

We will conduct an explanatory, sequential mixed methods study (Fig. 1). Nested within the larger FICare cRCT, we will examine the breastfeeding self-efficacy scores of participants in the intervention arm of the study. Using maximum variation sampling, we will select a sub-sample of mothers demonstrating the highest and lowest breastfeeding self-efficacy change scores during their infants' hospital stay. We will conduct a semi-structured telephone interview to explore maternal experiences of infant feeding during hospitalization. We will then use these experiences to explain how, or if, FICare informs maternal breastfeeding self-efficacy and resultant breastmilk feeding rates in mothers of moderate and late preterm infants. We adhered to the Standard Protocol Items: Recommendations for Interventional 


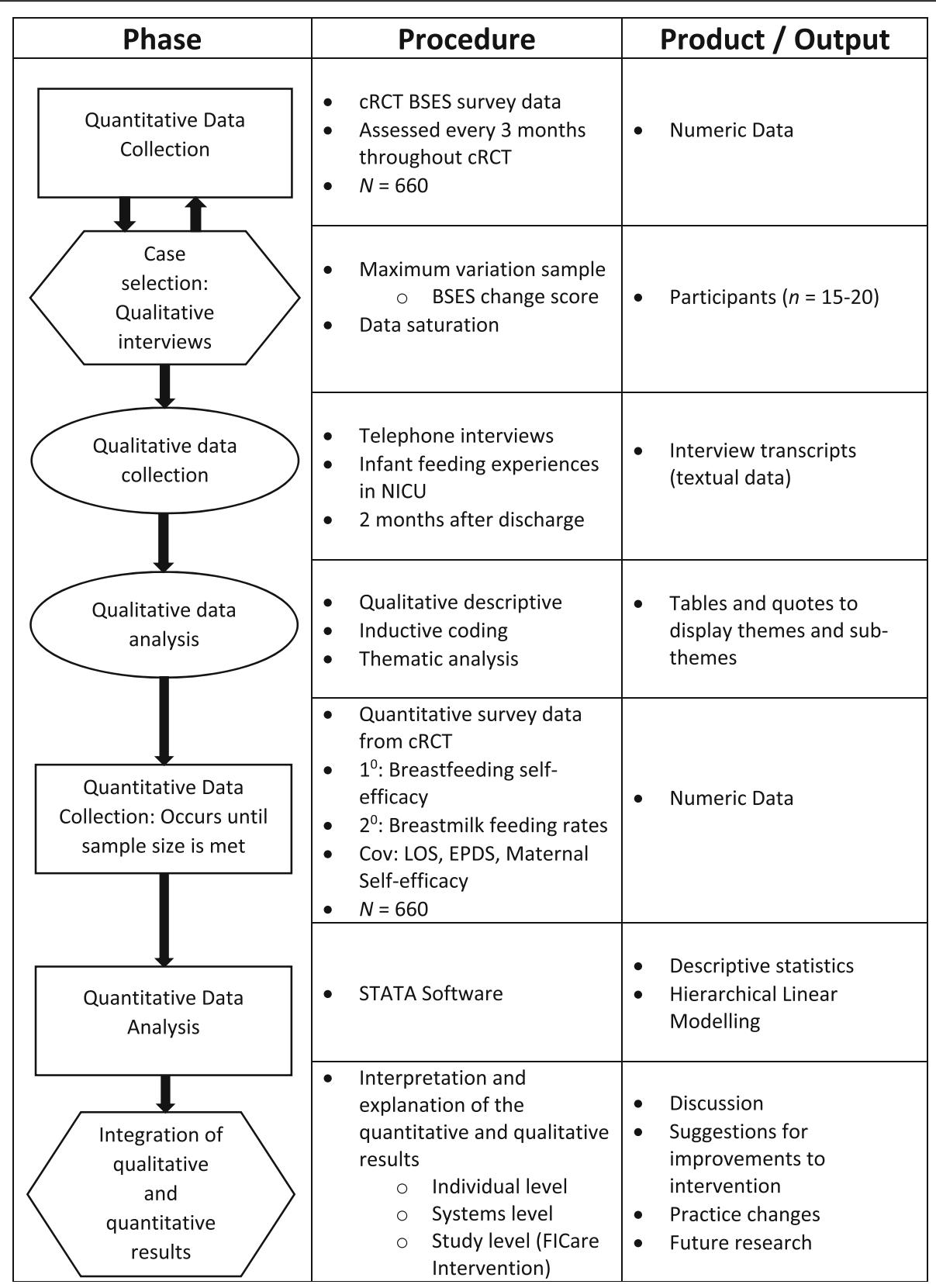

Fig. 1 Study flow diagram. Overview of the study design. Rectangles depict quantitative phase, ovals depict qualitative phases, and hexagons depict integration phases. Abbreviations: cluster randomized control trial (CRCT), Breastfeeding Self-efficacy Scale (BSES), covariates (Cov), length of stay (LOS), Edinburgh Postnatal Depression Scale (EPDS)

Trials (SPIRIT) guidelines in the design of the protocol (Additional file 1) [31].

\section{Rationale for mixed methods design}

There is a call to develop breastfeeding research methodologies that embrace "interpretation from the social sciences" rather than strictly relying on quantitative measures of breastmilk feeding rates [32]. Unilaterally implementing one of the two dominant research paradigms will not fully honour the complexity of breastfeeding self-efficacy and breastmilk feeding within the NICU [33]. Utilizing quantitative methodologies to measure breastfeeding self-efficacy and breastmilk feeding rates and qualitative methodologies to explore infant feeding experiences will allow for our research objective to be explored from multiple perspectives [33]. By exploring maternal experiences associated with feeding moderate and late preterm infants while in the NICU, we can better understand if FICare is a successful 
model of care to improve maternal breastfeeding self-efficacy and breastmilk feeding rates. Semi-structured interviews will allow for intimate insights from mothers to emerge that will provide substantive content as well as individual experiential data [34, 35]. Further, examining both the quantitative and qualitative components of breastfeeding self-efficacy will provide an enhanced understanding of breastfeeding self-efficacy results and resultant infant feeding outcomes.

\section{FICare cluster randomized controlled trial}

All level II NICUs in the province of Alberta, Canada ( $N=$ $10)$ will be randomized into a standard care control group $(n=5)$ or an intervention group $(n=5)$. Each intervention site will have specially trained registered nurse super-users who are responsible to recruit participants, deliver the intervention, and collect data. To ensure intervention fidelity, we will conduct site visits four times per year to assess adherence and compliance with the intervention components. We will also monitor for activities (e.g. policy changes, guidelines, unit practices) that may influence implementation of FICare.

\section{FICare model}

FICare is a model of care that actively supports families to participate in the care of their infant and was originally introduced to Canada at the Mount Sinai Hospital level III NICU in Toronto, Ontario [36]. The intervention originated from the Humane Neonatal Care model developed in Tallinn, Estonia, where parents actively participated in the care of their infants, while nurses and psychologists provided education and support [36]. Existing research regarding the effectiveness of FICare is limited to level III NICUs. A recent cRCT examining the impact of FICare in level III NICUs found that infants exposed to the FICare intervention were significantly more likely to be exclusively breastmilk feeding at discharge (279 [70\%] of 396) compared to those receiving standard care (394 [63\%] of 624; $p=0.016)$ [37]. As such, it is important to determine if the FICare model is similarly effective for improving breastmilk feeding outcomes in moderate and late preterm infants admitted to a level II NICU.

\section{FICare intervention}

Families participating in the FICare study will be required to spend a minimum of six hours per day, or approximately three feeding times, at the NICU. Nurses will support and educate mothers and fathers in their parenting role with a focus on actively involving them in the care of their infants while in the NICU. Parents will share in the care of their infant(s) as soon as they are able, starting with simple tasks, such as skin-to-skin contact and diapering, and progressing to more complex tasks, such as feeding. FICare involves three main components (Fig. 2): (i) information sharing, (ii) parent education (including parent-education pathways and specially designed apps), and (iii) parent support. Information sharing is bidirectional and involves parents verbally reporting on their infants' progress at daily bedside multidisciplinary rounds and actively contributing to discussions about the plan of care. Parent support will involve one-on-one discussions with veteran parents. Veteran parents (those who have previously had an infant in the level II NICU environment) will provide practical advice, guidance, and support to parents in the FICare study. Finally, parent education will include standardized, evidence informed parent education delivered individually or in group settings.

\section{FICare context - Level II NICUs}

In Alberta, infants admitted to a level II NICU are generally born after 32 weeks, weighing more than 1500 g. Nutritionally, these infants tend to be on total parenteral nutrition, receiving gavage feeds, or full oral feeds, depending on their developmental maturity [7]. All 10 level II NICUs in Alberta formally support the provision of mother's own milk as the first choice for infant feeding [38]. While there is no formalized breastfeeding education requirement [39] for practitioners working in NICUs, all mothers have access to International Board Certified Lactation Consultants. Visitation models vary among the 10 sites, with most NICUs allowing parents unrestricted 24-h access to their infant. There is a mix of open and closed ward models, with five of the NICUs planning to transition to single room wards during the timeline of the FICare study.

\section{FICare CRCT inclusion criteria}

The FICare cRCT will include mothers of preterm infants born between $32^{0 / 7}$ and $34^{6 / 7}$ weeks GA, admitted to a level II NICU in Alberta, who speak, read, and write English. To ensure an adequate dose of the intervention, infants must have a minimum NICU stay of 5 days. Infants born greater than 35 weeks GA were excluded from the study as they would not meet the minimum 5-day requirement if discharged around 36 weeks GA. We will exclude mothers of infants with social risk that may interfere with their ability to engage in FICare, and infants with a severe congenital abnormality or chromosomal anomaly, or receiving palliative care.

\section{FICare sample size}

We based sample size estimates on the primary outcome, length of stay. Due to an anticipated skewed distribution for length of stay, we used a natural logarithm transformation to calculate sample size [40]. To achieve a power of 0.80 , we need to recruit 181 mothers into each group for the primary outcome of length of stay; and 211 to achieve a power of 0.9. In 2014, there were 1030 moderate and late preterm infants admitted to a level II NICU in Alberta. To account for a response rate of $80 \%$ [36], attrition, and infants with a 


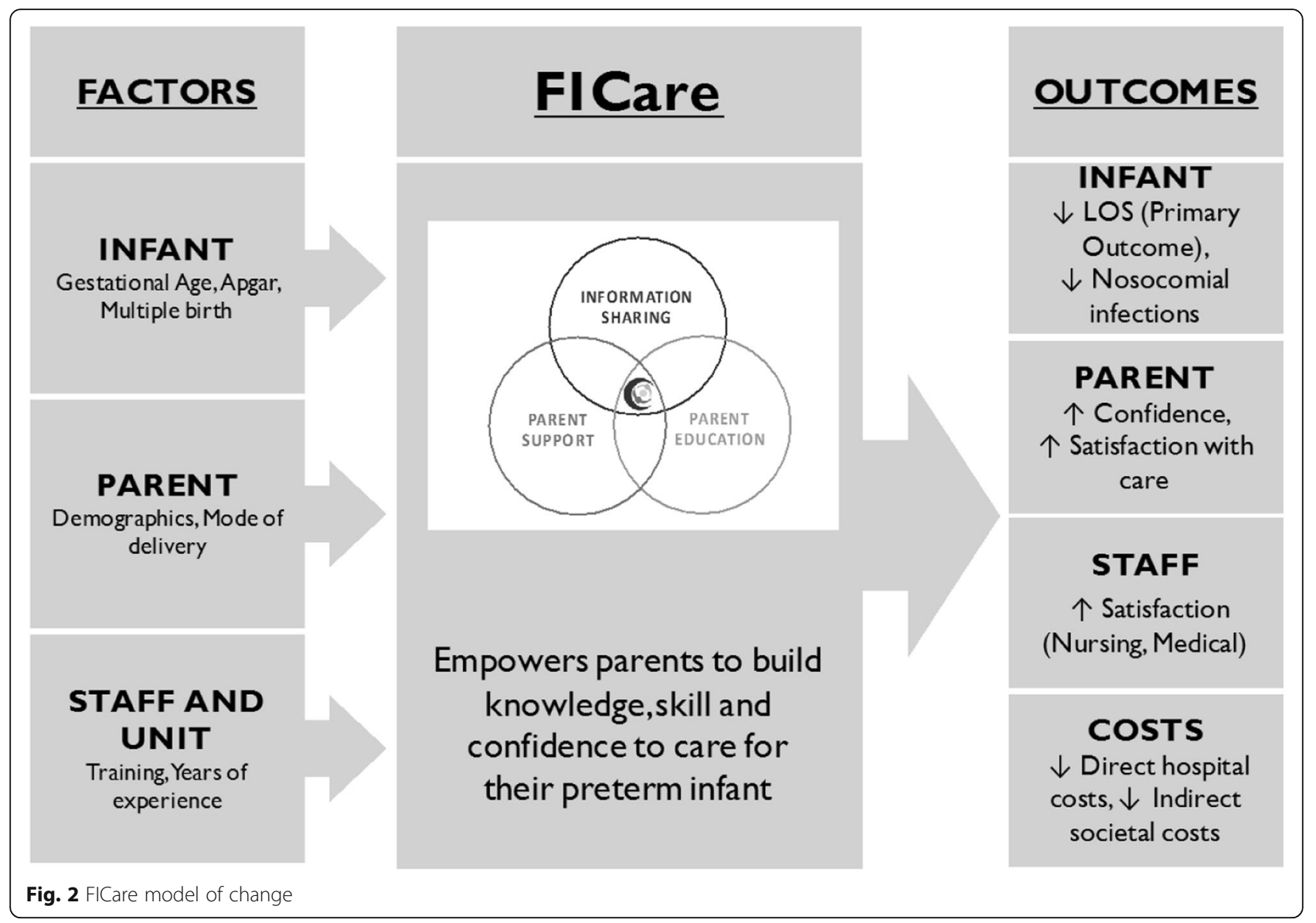

length of stay of $\leq 5$ days (6.08\%), we will approach 824 potential participants over the 30-month recruitment period. This will also ensure that the sample size, 330 per group, is sufficient to assess secondary outcomes and to provide the qualitative sample for the study.

\section{FICare recruitment}

Within $72 \mathrm{~h}$ of admission to the level II NICU, nurses will inform mothers about the study. If interested, a FICare super-user (a nurse specially trained in the FICare model) will screen mothers for eligibility, answer questions about the study, obtain informed consent, and administer the baseline questionnaire. As this is a cRCT, infants of mothers at the intervention sites who do not wish to participate in the study will receive the same hospital care as infants whose mothers are participating in the study. The present study will be nested within the larger FICare clinical trial (Fig. 3).

\section{Quantitative phase}

\section{Procedures}

Online questionnaires will be administered to mothers at two time points in the study (Table 1). The first set of questionnaires will be administered at enrolment; the second set about $24 \mathrm{~h}$ before the infant is discharged from the NICU.

\section{Measurement}

Breastfeeding self-efficacy will be measured using the modified Breastfeeding Self-efficacy Scale - short form (BSES-SF; Table 1) for mothers of ill and preterm infants [20]. The BSES-SF will only be administered to mothers who are breastfeeding, expressing their own breastmilk, attempting to breastfeed, or are planning to breastfeed. Mothers who have weaned or are not planning to breastfeed will not complete the BSES-SF. However, infant feeding rates will be captured for all infants in the study. BSES-SF data will be assessed quarterly throughout the quantitative data collection period and will inform purposive sampling for the qualitative phase of the study. The remaining quantitative data will be assessed upon completion of quantitative data collection and completion of qualitative data analysis.

The Labbok and Krasovek classification system [41] will be used to assess infant feeding rates at admission and discharge (Table 1). Maternal recall is frequently used as an effective method to collect infant feeding data 
Main Study: Family Integrated Care in Level II NICUs: An Innovative Program for Alberta

- Primary Outcome - Length of Stay - 10 Level II NICUs: 5 Intervention / 5 Control

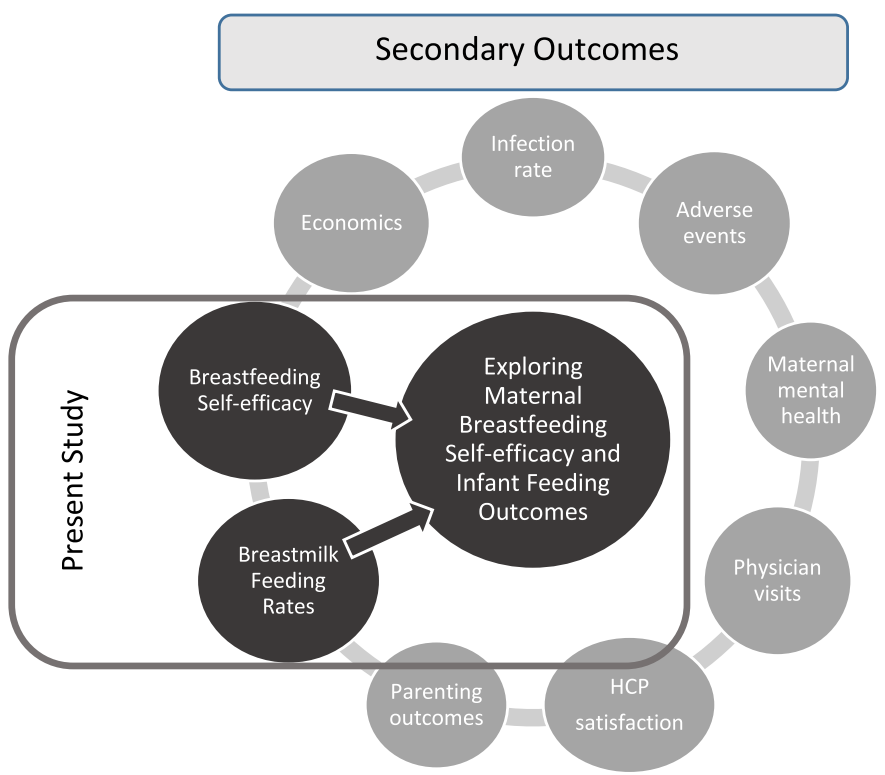

Fig. 3 Situation of present study within larger FICare clustered randomised control trial

[42]. Maternal recall will be used to assess infant feeding over the previous $24 \mathrm{~h}$ and will be classified as:

i. Exclusive breastmilk feeding - 100\% of feeds were breastmilk (including expressed breastmilk, donor human milk and additives)

ii. Mostly breastmilk - $75 \%$ of feeds were breastmilk (including expressed breastmilk, donor human milk and additives)

iii. Partial breastmilk feeding - 50\% of feeds were breastmilk (including expressed breastmilk, donor human milk and additives)

iv. Minimal breastmilk - 25\% of feeds were breastmilk (including expressed breastmilk, donor human milk and additives).

v. No breastmilk feeding - baby is not receiving any breastmilk

vi. nil per os (NPO) or nothing by mouth

The infant feeding questions will be predicated by $i$ ) is your baby receiving any human donor milk, and ii) is your baby receiving any additives to your breastmilk to help them grow?

\section{Data management}

We will collect data electronically and data will be stored on secure servers. Quantitative data will be managed as per Benzies et al. [30]. Upon completion of analysis, we will store data with the PolicyWise Secondary Analysis to Generate Evidence (SAGE; formerly the Child Data Centre of Alberta) database. This will help to facilitate data access by other qualified researchers.

\section{Data analysis}

Statistical analysis of the quantitative results will be performed using Stata Data Analysis and Statistical Software. The primary outcome of the statistical analysis is to determine if a difference exists in: i) breastfeeding self-efficacy scores (BSES-SF) and ii) breastmilk feeding rates, between the control and intervention groups. Results from the BSES-SF will be treated as continuous data whereas results from breastmilk feeding rates will be treated as categorical data [20,43]. Characteristics of participants and scores on scales will be presented as descriptive statistics (means, frequencies, and percentages). We will use an omnibus test (Hotelling's t-tests and Chi square) to assess for baseline differences between intervention and control groups on socio-demographic and 
Table 1 Outcome measures and potential covariates with breastfeeding self-efficacy and breastfeeding outcomes

\begin{tabular}{lll}
\hline Measure & Time point & Description \\
\hline Primary outcome & & \\
$\begin{array}{ll}\text { Modified Breastfeeding Self-Efficacy } & \text { Baseline; Discharge }\end{array}$ & $\begin{array}{l}\text { 18-item scale validated for mothers of ill and/or } \\
\text { preterm infants. Assesses a mother's confidence } \\
\text { Scale - Short Form [20] }\end{array}$ & $\begin{array}{l}\text { in her ability to breastfeed. Internal consistency } \\
(0.88) \text { is high. }\end{array}$
\end{tabular}

Secondary outcome

Breastmilk feeding

Co-variates and potential confounders

Parental Stressor Scale: NICU [62]

Baseline; Discharge

Edinburgh Postnatal Depression Scale [65]

State-Trait Anxiety Inventory [67]

Baseline; Discharge

Perceived Maternal Parenting Self-Efficacy scale [68]

Baseline; Discharge

Baseline; Discharge
Labbok and Krasovek [41] classification system, modified to include additives and fortification. 24-h maternal recall.

50-item scale that captures parental perceptions of stress in the NICU: (1) sights and sounds; (2) appearance and behaviour of the infant; (3) impact on the parental role and relationship with the infant; and (4) parental relationship and communications with staff. Internal consistency (0.89 to 0.94 for the total scale) and test-retest (0.87) reliabilities are high.

Potential confounding: Decreasing maternal anxiety and stress may have a physiologic impact on breastmilk production $[63,64]$

The most commonly used pre- and post-natal depression screener validated for mothers. Consists of 10 items and has a sensitivity of 0.86 and specificity of 0.78 , with a positive predictive value of $73 \%$. Potential confounding: Successful breastfeeding is predictive of lower maternal depressive symptomology. Depressive symptoms may be predictive of reduced breastfeeding rates [66].

40-item scale that captures dispositional/trait anxiety (20 items) and current state anxiety (20 items). Internal consistency (0.86 to 0.95$)$ and test-retest ( 0.73 to 0.86 ) reliabilities are high. Scores on the STAI and PSS: NICU are correlated [62].

Potential confounding: Mothers who demonstrate high rates of anxiety or depressive symptomology routinely have lower breastfeeding rates than mothers that do not [66].

20-item measure of parenting self-efficacy validated for mothers of preterm infants. Captures maternal perceptions of ability to (1) give basic care; (2) elicit change in infant behaviour; (3) recognize infant behaviour; and (4) judge interactions with her infant. Exploratory factor analysis confirms four factors; internal consistency (0.91) and test-retest (0.96) reliabilities are high.

Potential confounding: Concurrent validity between general self-efficacy and BSES-SF [22].

${ }^{a}$ At admission, both State and Trait forms are completed; at discharge only State form is completed. Adapted from Benzies et al. [30]

health characteristics, breastfeeding self-efficacy, and breastmilk feeding rates.

To test if there is a difference in breastfeeding self-efficacy and breastmilk feeding rates between the intervention and control groups, we will use Hierarchical Linear Modeling (HLM; also known as multilevel modeling or mixed-effect modeling) and Hierarchical General Linear Modeling (HLGM), respectively. This study is nested in a larger cRCT and there is a potential for variance in care delivery at each of the NICU sites. As such, there are multiple levels of data that need to be considered to accurately assess the effectiveness of the intervention on breastfeeding self-efficacy and breastmilk feeding rates. By simultaneously investigating the relationships between the different levels of data, HLM and HGLM analysis can account for variance among variables at different levels [44]. The HLM approach will allow for the two observations (admission and discharge from the NICU) by treating each participant's breastfeeding self-efficacy score and breastmilk feeding rate at 
each assessment point as single data points [44]. There will be two levels of analysis in the data: Level 1 will refer to the outcome variables of breastfeeding self-efficacy and breastmilk feeding rates; while Level 2 will be the maternal or subject effect. We will enter the subject effect into the model as a random effect to capture within and between-subject variation [44]. The group effect of intervention/control will be entered as fixed effect to determine if the intervention is effective.

\section{Qualitative phase}

We will employ a qualitative descriptive exploration [45] and thematic analysis [46] of maternal experiences with the FICare intervention and infant feeding in the NICU. Qualitative description involves low-inference interpretation of the data [45]. Thematic analysis is used by researchers as a technique to analyse data in qualitative descriptive studies $[47,48]$. Using thematic analysis will allow us to examine and compare different perspectives of infant feeding experiences while in the NICU, as well as help to generate unanticipated insights [48].

\section{Sampling}

Using a variation of purposive, maximum variation sampling $[49,50]$, we will select mothers who experience high positive mean differences in their breastfeeding self-efficacy scores between NICU admission and discharge. To capture experiences that may have worked to detract from maternal breastfeeding self-efficacy, we will also select mothers with high negative mean differences. Assuming a normal distribution, we will sample from the top and bottom $20 \%$ of the change score distribution and sampling will continue until data saturation is achieved [50].

\section{Data collection}

We will conduct semi-structured telephone interviews (Additional file 2). The geographical dispersion of participants in the FICare study renders face-to-face interviews unfeasible, and telephone interviews will allow data collection to occur with minimal expenditure and time commitment. Telephone interviews are an effective and efficient approach to qualitative data collection and provide results similar to face-to-face interviews [51]. Telephone interviews may also help to reduce social desirability response bias and interviewer effects, which may be more prevalent in face-to-face interviews [51]. Interviews will be conducted until informational redundancy is achieved and no new topics or concepts are emerging with additional interviews, with a projected sample size of 15 [52]. We will allow participants to guide the conversation, with minimal probing and redirection to maintain the content of the interview within the context of the themes requiring verification.

\section{Data analysis}

We will conduct thematic analysis within a constructionist framework, focusing on the sociocultural context and structural conditions (such as NICU policies, physical space and relationships with practitioners) that inform infant feeding experiences [46]. Employing theoretically driven coding may not fully capture maternal experiences with infant feeding while in the NICU. As such, we will use an inductive approach to thematic analysis $[46,53]$ to enable themes to be developed that are linked to the data and not to a previously determined theory. The inductive technique of data analysis will allow for codes and sub-codes to describe themes as they are observed in the data [54]. Inductively driven coding is constructed from the raw data and is interpreted by the researcher [53]. This may result in the development of themes that are not directly related to the pre-determined qualitative research question [53]. Coding will occur through a three-step process. The first step will be to read through transcripts to find codable moments that emerge from patterns in the data. Once the pattern is identified, we will move on to the second step of classifying or encoding the pattern by giving it a label [53]. Finally, once the data has been sufficiently coded and we have reached saturation, we will interpret the themes using thematic networks [55]. Selecting mothers based on their mean difference breastfeeding self-efficacy scores will situate breastfeeding self-efficacy as the criterion reference [53]. We will be able to compare-and-contrast themes that emerge from infant feeding experiences and identify observable differences between mothers with low and high breastfeeding self-efficacy change scores [53]. We will bracket our assumptions of the breastfeeding self-efficacy theory [56] and allow for themes to emerge as they are related to the concept of maternal experiences with infant feeding in the NICU. Bracketing is a reflexive process that involves preparation, action, evaluation, and systematic feedback [57] regarding thought processes and data analysis techniques throughout the coding process. Bracketing will be conducted through acknowledging our assumptions of, and affinity for, the breastfeeding self-efficacy theory and conducting reflexive journaling [56].

\section{Integration of quantitative and qualitative phases}

Methodologically, the qualitative sample emerges from the quantitative scores on BSES-SF and depends entirely on the analysis and sample selection strategy $[53,58]$. As such, the qualitative phase of the study is entirely dependent on the analysis of the quantitative breastfeeding self-efficacy results.

To fully benefit from the complementarity of the explanatory sequential design, explicit linkages must be made between the quantitative and qualitative results. [58]. Integration of the qualitative and quantitative 
results will be conducted in three stages. The first stage of integration will occur at the theoretical level and will relate the findings of the qualitative thematic analysis to the four sources of information in the breastfeeding self-efficacy theory [21, 59]. The breastfeeding self-efficacy theory forms the theoretical underpinnings of the study and provides a framework from which to integrate the quantitative and qualitative findings. This will provide a comprehensive understanding of how, or if, maternal experiences with infant feeding in the NICU work to inform breastfeeding self-efficacy. The second stage will examine the qualitative findings with respect to the system level. Themes regarding infant feeding experiences in relation to the NICU environment will be used to explain barriers and facilitators to provision of breastmilk or breastfeeding. The final stage of integration will focus on themes that are directly related to the FICare model of care and how these themes can influence infant breastmilk feeding rates. The second and third stages of integration will occur at the practical level, developing inferences regarding practices and models of care that may impact breastmilk feeding outcomes.

\section{Discussion}

Our proposed mixed methods study will assess if FICare is an effective care practice to improve breastfeeding self-efficacy and breastmilk feeding rates in mothers of moderate and late preterm infants. The findings will also contribute evidence to the limited body of knowledge regarding breastfeeding self-efficacy in the moderate and late preterm population. The qualitative data will allow us to elaborate, enhance, and clarify quantitative findings so that inferences can be drawn regarding the FICare intervention and meta-inferences can be made to the broader NICU population [60]. Hypothetically, these inferences may include structured recommendations regarding FICare that specifically address maternal breastfeeding self-efficacy or structural modifications to the NICU that make mothers feel more adept at providing breastmilk for their infant(s). Additionally, the explanatory sequential typology will allow us to examine the convergence, corroboration, and correspondence of breastfeeding self-efficacy results from the quantitative and qualitative findings [60].

This explanatory sequential study will not only serve to assess the effectiveness of the FICare intervention on breastfeeding self-efficacy, but will also provide an in-depth understanding of how the elements of FICare work to inform breastfeeding self-efficacy and subsequent breastmilk feeding rates. Improving breastfeeding self-efficacy and breastmilk feeding rates will provide increased evidence of the effectiveness of the FICare intervention. If effective, FICare can fundamentally change care delivery methods in Level II NICUs and may serve to improve breastmilk feeding outcomes in moderate and late preterm infants.

\section{Additional files}

Additional file 1: SPIRIT 2013 Checklist: Recommended items to address in a clinical trial protocol and related documents. (DOC $123 \mathrm{~kb}$ )

Additional file 2: Mother Interview Guide - Infant Feeding Experiences in the NICU. (DOCX $17 \mathrm{~kb})$

\section{Abbreviations}

BSES-SF: Breastfeeding self-efficacy scaleshort form; CRCT: Cluster randomized controlled trial; FICare: Family Integrated Care; GA: Gestational age;

NICU: Neonatal intensive care unit

\section{Acknowledgements}

The authors acknowledge the contributions of the Alberta FlCare in Level II NICU team, Pilar Zanoni for study coordination, and Arfan Afzal for statistical expertise.

\section{Funding \\ This research was funded by: Alberta Innovates - Health Solutions, Partnership for Innovation in Health Services Research (PRIHS) grant number 201400399, with in-kind support from Alberta Health Services and Faculty of Nursing, University of Calgary. The FICare study team has ultimate authority over all decisions related to the study, including publications. Meredith Brockway received PhD scholarships from the Alberta Strategy for Patient Oriented Research, the Alberta Children's Hospital Research Institute (ACHRI), and the University of Calgary; and Graduate studentships from the Faculty of Nursing at the University of Calgary.}

\section{Availability of data and materials}

FICare investigators will have access to the anonymized dataset for analyses. $\mathrm{MB}$ will use the data from this study to contribute to her graduate student thesis. Anonymized data generated from this study will be deposited in PolicyWise, Secondary Analysis to Generate Evidence (SAGE) for access by other qualified researchers [61].

\section{Authors' contributions}

Responsible for trial implementation and management KB and KA. Responsible for supervision and methodologic guidance of primary author $K B, K A$, EC. Responsible for ethics applications and reporting: $M B, K B$, and $K A$. Responsible for writing the first draft of the protocol manuscript: MB. Responsible for preparation and submission of manuscript MB. All authors have critically reviewed and approved the final version of the study protocol manuscript.

\section{Ethics approval and consent to participate}

Ethics approval was received from the Conjoint Health Research Ethics Board, University of Calgary (REB 15-0067). Written informed consent will be obtained for all research participants.

Supporting documents (model consent form), protocol amendments, and processes of consent or assent and confidentiality can be found at Family Integrated Care (FICare) in Level II Neonatal Intensive Care Units: study protocol for a cluster randomized controlled trial [30].

Consent for publication

Not applicable.

Competing interests

The authors declare that they have no competing interests. 


\section{Publisher's Note}

Springer Nature remains neutral with regard to jurisdictional claims in published maps and institutional affiliation.

\section{Author details}

${ }^{1}$ Faculty of Nursing, University of Calgary, Calgary, AB, Canada. ${ }^{2}$ Department of Pediatrics, Faculty of Medicine and Dentistry, University of Alberta, Edmonton, $\mathrm{AB}$, Canada.

Received: 2 February 2018 Accepted: 4 June 2018

Published online: 06 July 2018

\section{References}

1. Canadian Institutes for Health Information. Health Indicators 2012. Ottawa: CIHI; 2012. https://secure.cihi.ca/free_products/health_indicators_2012_en. pdf. Accessed 11 Nov 2017

2. World Health Organization. Born too soon. The global action report on preterm birth. Geneva: WHO; 2012. http://apps.who.int/iris/bitstream/handle/10665/44864/ 9789241503433_eng.pdf;isessionid=EOF5AA36E702BADC39COFC 19B82E2C31? sequence $=1$. Accessed 11 Nov 2017

3. Engle W, Tomashek K, Wallman C. "Late-preterm" infants: a population at risk. Pediatrics. 2007;120(6):1390-401.

4. Blencowe $H$, Cousens S, Oestergaard MZ, Chou D, Moller A-B, Narwal R, Adler A, et al. National, regional, and worldwide estimates of preterm birth rates in the year 2010 with time trends since 1990 for selected countries: a systematic analysis and implications. Lancet. 2012;379(9832):2162-72.

5. Morken $\mathrm{N}-\mathrm{H}$. Preterm birth: new data on a global health priority. Lancet. 2012;379(9832):2128-30.

6. Natarajan G, Shankaran S. Short- and long-term outcomes of moderate and late preterm infants. Am J Perinatol. 2016;33(03):305-17.

7. Barfield WD, Papile L-A, Baley JE, Benitz W, Cummings J, Carlo WA, et al. Levels of neonatal care. Pediatrics. 2012;130(3):587-97.

8. Lucas $\mathrm{R}$, Smith RL. When is it safe to initiate breastfeeding for preterm infants? Adv Neonatal Care. 2015;15(2):134-41.

9. Kim J, Unger S. Human milk banking. Paediatrics Child Health. 2010;15(9): 595-8.

10. Victora CG, Bahl R, Barros AJD, França GVA, Horton S, Krasevec J, et al. Breastfeeding in the 21st century: epidemiology, mechanisms, and lifelong effect. Lancet. 2016:387(10017):475-90.

11. Zhou J, Shukla W, John D, Chen C. Human milk feeding as a protective factor for retinopathy of prematurity: a meta-analysis. Pediatrics. 2015;136(6): e1576-86.

12. World Health Organization. Guidelines on optimal feeding of low birthweight infants in low- and middle-income countries. Geneva: WHO; 2011. http://www.who.int/maternal_child_adolescent/documents/infant_feeding_ low_bw/en/. Accessed 11 Nov 2017

13. Ross ES, Browne JV. Feeding outcomes in preterm infants after discharge from the neonatal intensive care unit (NICU): a systematic review. Newborn Infant Nurs Rev. 2013;13(2):87-93.

14. Goyal NK, Attanasio LB, Kozhimannil KB. Hospital care and early breastfeeding outcomes among late preterm, early-term, and term infants. Birth. 2014:41(4):330-8

15. Callen J, Pinelli J. A review of the literature examining the benefits and challenges, incidence and duration, and barriers to breastfeeding in preterm infants. Adv Neonatal Care. 2005;5(2):72-88.

16. American Academy of Pediatrics. Family pediatrics: report of the task force on the family. Pediatrics. 2003;111(Suppl 2):1541-71.

17. Griffin T. Family-centered care in the NICU. J Perinat Neonatal Nurs. 2006; 20(1):98-102.

18. Gooding JS, Cooper LG, Blaine Al, Franck LS, Howse JL, Berns SD. Family support and family-centered care in the neonatal intensive care unit: origins, advances, impact. Semin Perinatol. 2011;35(1):20-8.

19. Staub K, Baardsnes J, Hébert N, Hébert M, Newell S, Pearce R. Our child is not just a gestational age. A first-hand account of what parents want and need to know before premature birth. Acta Paediatr. 2014;103(10):1035-8.

20. Wheeler BJ, Dennis CL. Psychometric testing of the modified breastfeeding self-efficacy scale (short form) among mothers of ill or preterm infants. J Obstet Gynecol Neonatal Nurs. 2013;42(1):70-80.

21. Dennis CL. Theoretical underpinnings of breastfeeding confidence: a selfefficacy framework. J Hum Lact. 1999;15(3):195-201.
22. Dennis $\mathrm{CL}$, Faux S. Development and psychometric testing of the breastfeeding self-efficacy scale. Res Nurs Health. 1999;22(5):399-409.

23. Dennis CL. The breastfeeding self-efficacy scale: psychometric assessment of the short form. J Obstet Gynecol Neonatal Nurs. 2003;32(6):734-44.

24. Schwarzer R, Fuchs R. Self-efficacy and health behaviours. In: Conner M, Norman $\mathrm{P}$, editors. Predicting health behaviour: research and practice with social cognition models. New York: Open University Press; 1996. p. 163-96.

25. Bandura A. Self-efficacy: toward a unifying theory of behavioral change. Psychol Rev. 1977;84(2):191-215.

26. Brockway M, Benzies K, Hayden KA. Interventions to improve breastfeeding self-efficacy and resultant breastfeeding rates: a systematic review and meta-analysis. J Hum Lact. 2017;33(3):486-99.

27. Skouteris $H$, Nagle $C$, Fowler $M$, Kent $B$, Sahota $P$, Morris $H$. Interventions designed to promote exclusive breastfeeding in high-income countries: a systematic review. Breastfeed Med. 2014;9(3):113-27.

28. Tuthill EL, McGrath JM, Graber M, Cusson RM, Young SL. Breastfeeding selfefficacy: a critical review of available instruments. J Hum Lact. 2015:32(1):35-45.

29. Gerhardsson E, Hildingsson I, Mattsson E, Funkquist E-L. Prospective questionnaire study showed that higher self-efficacy predicted longer exclusive breastfeeding by the mothers of late preterm infants. Acta Paediatr. 2018:107(5):799-805.

30. Benzies KM, Shah V, Aziz K, Isaranuwatchai W, Palacios-Derflingher L, Scotland J, et al. Family integrated care (FICare) in level II neonatal intensive care units: protocol of a pragmatic, cluster randomized controlled trial of clinical effectiveness and cost of integrating families into the care of moderate and late preterm infants. BMC Trials. 2017;18:467.

31. Chan A, Tetzlaff JM, Altman DG, Laupacis A, Gotzsche PC, Krleza-Jeric K, et al. SPIRIT 2013 statement: defining standard protocol items for clinical trials. Ann Intern Med. 2013;158(3):200-7.

32. Spencer R. Ontological perspectives of breastfeeding. Br J Midwifery. 2007; 15(12):779-83.

33. Johnson RB, Onwuegbuzie AJ, Turner LA. Toward a definition of mixed methods research. J Mixed Methods Res. 2007;1(2):112-33.

34. Ryan F, Coughlan M, Cronin P. Interviewing in qualitative research: the oneto-one interview. Int J Ther Rehabil. 2009;16(6):309-14.

35. DiCicco-Bloom B, Crabtree BF. The qualitative research interview. Med Educ 2006;40(4):314-21

36. O'Brien K, Bracht M, Macdonell K, McBride T, Robson K, O'Leary L, et al. A pilot cohort analytic study of family integrated Care in a Canadian neonatal intensive care unit. BMC Pregnancy Childbirth. 2013;13(Suppl 1):S12.

37. O'Brien K, Robson K, Bracht M, Cruz M, Lui K, Alvaro R, et al. Effectiveness of family integrated care in neonatal intensive care units on infant and parent outcomes: a multicentre, multinational, cluster-randomised controlled trial. Lancet Child Adolescent Health. 2018;2(4):245-54.

38. Alberta Health Services. Oral feeding: neonatal intensive care, Calgary Zone. Calgary: Alberta Health Services; 2016. p. 30. https://extranet.ahsnet.ca/ teams/policydocuments/1/clp-calgary-childrens-health-neonatologynutrition-oral-feeding-2-o-2.pdf. Accessed 12 Apr 2018

39. World Health Organization, United Nations Children's Fund. Baby-friendly hospital initiative: revised, updated and expanded for integrated care. Section 3: breastfeeding promotion and support in a baby-friendly hospital. Geneva: WHO; 2009.

40. Sedgwick P. Log transformation of data. BMJ. 2012;345:e6727.

41. Labbok M, Krasovec K. Toward consistency in breastfeeding definitions. Stud Fam Plan. 1990:21(4):226-30.

42. Launer LJ, Forman MR, Hundt GL, Sarov B, Chang D, Berendes HW, et al. Maternal recall of infant feeding events is accurate. J Epidemiol Community Health. 1992:46(3):203-6.

43. Jamieson S. Likert scales: how to (ab)use them. Med Educ. 2004;38(12):1217-8.

44. Woltman $\mathrm{H}$, Feldstain A, MacKay JC, Rocchi M. An introduction to hierarchical linear modeling. Tutor Quant Methods Psychol. 2012;8(1):52-69.

45. Sandelowski M. Whatever happened to qualitative description? Res Nurs Health. 2000;23(4):334-40.

46. Braun V, Clarke V. Using thematic analysis in psychology. Qual Res Psychol. 2006:3(2):77-101.

47. Vaismoradi M, Turunen $H$, Bondas $T$. Content analysis and thematic analysis: implications for conducting a qualitative descriptive study. Nurs Health Sci. 2013:15(3):398-405.

48. Nowell LS, Norris JM, White DE, Moules NJ. Thematic analysis: striving to meet the trustworthiness criteria. Int J Qual Methods. 2017:16(1). https://doi. org/10.1177/1609406917733847. 
49. Patton MQ. Variety of qualitative inquiry frameworks: paradigmatic, philosophical, and theoretical orientations. In: Qualitative research \& evaluation methods: integrating theory and practice. 4th ed. Thousand Oaks: SAGE; 2014. p. 85-168.

50. Kuzel AJ. Sampling in qualitative inquiry. In: Crabtree BF, Miller WL, editors. Doing qualitative research. 2nd ed. Thousand Oaks: SAGE; 1999. p. 33-46.

51. Carr ECJ, Worth A. The use of the telephone interview for research. Nurs Times Res. 2001;6(1):511-24.

52. Sandelowski M. Theoretical saturation. In: Given LM, editor. The Sage encyclopedia of qualitative research methods, $2^{\text {nd }}$ edn. Thousand Oaks: SAGE; 2008. p. 875-6.

53. Boyatzis RE. Developing themes and a code using the inductive method: an example using life stories. In: Transforming qualitative information: thematic analysis and code development. Thousand Oaks: SAGE; 1998. p. 67-98.

54. Fereday J, Muir-Cochrane E. Demonstrating rigor using thematic analysis: a hybrid approach of inductive and deductive coding and theme development. Int J Qual Methods. 2008;5(1):80-92.

55. Attride-Stirling J. Thematic networks: an analytic tool for qualitative research. Qual Res. 2001;1(3):385-405.

56. Fischer $C$. Bracketing in qualitative research: conceptual and practical matters. Psychother Res. 2009;19(4-5):583-90.

57. Ahern KJ. Ten tips for reflexive bracketing. Qual Health Res. 1999;9(3):407-11.

58. Creswell JW, Plano Clark VL. Analyzing and interpreting data in mixed methods research. In: Designing and conducting mixed methods research. 2nd ed. Thousand Oaks: SAGE; 2011. p. 203-50.

59. Kingston D, Dennis CL, Sword W. Exploring breast-feeding self-efficacy. J Perinat Neonatal Nurs. 2007;21(3):207-15.

60. Greene JC, Caracelli VJ, Graham WF. Toward a conceptual framework for mixed-method evaluation designs. Educ Eval Policy Anal. 1989;11(3):255-74.

61. Policy Wise for Children and Families. SAGE - Secondary Analysis to Generate Evidence. https:/policywise.com/initiative/sage/. Accessed 11 Nov 2017.

62. Miles MS, Funk SG, Carlson J. Parental stressor scale: neonatal intensive care unit. Nurs Res. 1993;42(3):148-52.

63. Zanardo V, Gambina I, Begley C, Litta P, Cosmi E, Giustardi A, et al. Psychological distress and early lactation performance in mothers of late preterm infants. Early Hum Dev. 2011;87(4):321-3.

64. Dewey KG. Maternal and fetal stress are associated with impaired lactogenesis in humans. J Nutr. 2001;131(11):3012S-5S.

65. Cox JL, Holden JM, Sagovsky R. Detection of postnatal depression: development of the 10-item Edinburgh postnatal depression scale. Br J Psychiatry. 1987;150:782-6.

66. Hahn-Holbrook J, Haselton MG, Dunkel Schetter C, Glynn LM. Does breastfeeding offer protection against maternal depressive symptomatology? Arch Womens Ment Health. 2013;16(5):411-22.

67. Spielberger CD, Gorsuch RL, Lushene RE. Test manual for the state-trait anxiety inventory. Palo Alto: Consulting Psychologists Press; 1970

68. Barnes CR, Adamson-Macedo EN. Perceived maternal parenting self-efficacy (PMP S-E) tool: development and validation with mothers of hospitalized preterm neonates. J Adv Nurs. 2007;60(5):550-60.

\section{Ready to submit your research? Choose BMC and benefit from:}

- fast, convenient online submission

- thorough peer review by experienced researchers in your field

- rapid publication on acceptance

- support for research data, including large and complex data types

- gold Open Access which fosters wider collaboration and increased citations - maximum visibility for your research: over $100 \mathrm{M}$ website views per year

At BMC, research is always in progress.

Learn more biomedcentral.com/submissions 